\title{
Procesos de autorregulación del aprendizaje y desempeño académico en estudiantes de pregrado bajo la modalidad virtual
}

\section{Processes auto regulation of learning an academic performance in undergraduate students under virtual learling}

DOI: http://dx.doi.org/10.17981/cultedusoc.11.2.2020.12

Recibido: 1 de abril de 2020 Aceptado 26 de junio de 2020 Publicado: 09 de julio de 2020

\author{
Claudia Tatiana Mora Yate \\ Corporación Universitaria Minuto de Dios. Bogotá, D.C. (Colombia) \\ claudiamoray8@gmail.com \\ Jenny Consuelo Mahecha Escobar \\ Corporación Universitaria Minuto de Dios. Bogotá, D.C. (Colombia) \\ jmahecha@uniminuto.edu \\ Francisco Conejo Carrasco \\ Corporación Universitaria Minuto de Dios. Bogotá, D.C. (Colombia) \\ francisco.conejo@uniminuto.edu
}

Para citar este artículo:

Mora, C.. Mahecha, J. y Carrasco, F. (2020). Procesos de autorregulación del aprendizaje y desempeño académico en estudiantes de pregrado bajo la modalidad virtual. Cultura, Educación y Sociedad, 11(2). 191-206. DOI: http://dx.doi.org/10.17981/ cultedusoc.11.2.2020.12

\section{Resumen}

En el contexto educativo virtual, los procesos de autorregulación del aprendizaje juegan un papel muy importante por cuanto, son una herramienta que permite al estudiante alcanzar sus logros académicos. En este artículo se analiza la relación entre los procesos de autorregulación del aprendizaje y el desempeño académico de los estudiantes de licenciatura en pedagogía infantil en la asignatura de antropología social y cultural en una Universidad privada. Este estudio se desarrolló bajo un enfoque metodológico de tipo cualitativo, diseño descriptivo con un grupo de interés conformado por 10 estudiantes y 2 docentes. Los resultados indican que los factores de autorregulación del aprendizaje potenciadores del desempeño académico son la motivación, las estrategias pedagógicas y el autoconcepto, asimismo, se logró determinar que, aunque los estudiantes cuentan con procesos de autorregulación del aprendizaje estos se deben potenciar.

Palabras clave: Autorregulación; motivación; aprendizaje autónomo; rendimiento académico; estrategias de aprendizaje; Aprendizaje virtual

\section{Abstract}

In the virtual educational context, the processes of self-regulation of learning play a very important role in that they are a tool that enables the student to achieve academic achievement. This article analyzes the relationship between the self-regulatory learning processes and the academic performance of undergraduate students in child pedagogy in the subject of social and cultural anthropology at a private university. This study was developed under a qualitative methodological approach, descriptive design with an interest group made up of 10 students and 2 teachers. The results indicate that the self-regulating factors of learning that enhance academic performance are motivation, pedagogical strategies and self-concept. Likewise, it was possible to determine that, although the students have self-regulating learning processes, these should be promoted.

Keywords: Auto regulation; motivation; autonomous learning; academic performance; learning stategies, virtual learning 


\section{INTRODUCCIÓN}

En el contexto educativo contemporáneo, específicamente en la educación superior bajo la modalidad virtual, existen una serie de requerimientos y habilidades con las cuales debe contar cada estudiante, para su trascurso formativo profesional, de acuerdo a ello, dentro de la enseñanza aprendizaje, la autorregulación del aprendizaje y los procesos que esta incluye tales como la motivación, autoconcepto, autoeficacia entre otros, juegan un papel importante y de gran relevancia, puesto que son considerados como una herramienta la cual le permite al estudiante alcanzar sus logros académicos. Es decir, que la autorregulación del aprendizaje puede considerarse como un constructo psicológico a partir del cual el estudiante es autónomo de su aprendizaje y puede configurar sus actividades y para alcanzar sus objetivos y metas establecidas (Hernández y Camargo, 2017).

Dentro de la investigación pedagógica contemporánea se evidencia la necesidad de potencializar los procesos de autorregulación del aprendizaje en cada estudiante logrando así que este sea cada día más autónomo con su desarrollo pedagógico, es por ello que se deben incluir y potencializar estrategias de aprendizaje no solo mediante las clases, sino a través de todo el material disponible en la plataforma, lo cual permite al estudiante desenvolverse de manera autónoma no solo en el contexto académico, sino personal, social y profesional. Agregando a lo anterior, este estudio explora, la relación entre los procesos de autorregulación del aprendizaje y el desempeño académico de los estudiantes de licenciatura en pedagógica infantil bajo la modalidad virtual de una universidad privada en la asignatura de antropología social y cultural, para dar respuesta se realizó la siguiente revisión literaria.

\section{Fundamentos teóRICOS}

En el contexto académico, se han creado diversas discusiones con relación al concepto de Autorregulación del aprendizaje, este es asociado al desarrollo o curso mediante los cuales las personas adquieren conocimientos en los diferentes escenarios, principalmente en el académico; a partir de ello, la autorregulación se ha investigado desde diversas áreas tales como la pedagogía y la psicología, trayendo consigo teorías, aportes teóricos, investigaciones e instrumentos para la evaluación y medición de la misma.

En relación a lo anterior, Azevedo (citado en Moreno, 2015), afirma que el aprendizaje autorregulado en el estudiante se da mediante cinco fases; el primero de ellos es la planeación (objetivos, conocimiento y metas de memoria), seguido de monitoreo (análisis de lo que se aprende, auto cuestionamientos), posterior a ello, el uso de estrategias (selección de información, lectura, evaluación de contenidos), demanda y dificultad de tareas (planeación, control de aprendizaje) y finalmente la declaración de interés (interés por la actividad académica).

Por otro lado, De Acedo (2010) sostiene que la autorregulación del aprendizaje es un proceso propio del estudiante, el cual le permite ser consciente de su desarrollo académico y participar de una manera activa en este, a su vez se puede ver reflejada en el estudiante mediante tres factores tales como los cognitivos (propios del estudiante), conductuales y afectivos, estos tres se encuentran encaminados a una meta u objetivo pedagógico establecido. 
Con base en las definiciones anteriores, se puede evidenciar que la autorregulación del aprendizaje hace referencia a un suceso individual, que desarrolla el estudiante mediante su progreso pedagógico, donde juegan un papel importante los factores cognitivos y conductuales permitiendo un óptimo desarrollo del estudiante. Asimismo, el rol y quehacer del docente en el aula de clase o ambiente educativo hacen parte de dicho aprendizaje.

En el proceso de formación superior, los estudiantes deben identificar y proyectarse metas de aprendizaje, ya sean a corto o mediano plazo, a partir de ello desde la enseñanzaaprendizaje se debe realizar un proceso de acompañamiento, en el que el docente incursione diversas mediaciones pedagógicas, que le permitan crear una relación bidireccional con sus estudiantes y de esta manera potenciar la motivación de los mismos frente a factores cognitivos y conductuales para que el alumno logre llegar a la meta de manera satisfactoria y autorregulada en su proceso académico. De acuerdo con Klimenko y Alvares (2009) en la educación actual es importante fomentar en los estudiantes las competencias necesarias para contar con un aprendizaje autorregulado, por ello la importancia el manejo de las estrategias metacognitivas.

\section{Modelos teóricos sobre autorregulación}

Se abordó el modelo de Zimmernman, puesto que es considerado el más acertado en relación a esta idea de investigación, ya que, en su modelo teórico se considera a los estudiantes como activos y conscientes de su proceso de autorregulación, asimismo, contemplan las áreas cognitivas, motivacionales, conductuales y contextuales como fundamentales para el éxito académico del estudiante.

Modelo de Zimmerman: Para Zimmerman (1989), la autorregulación hace referencia a un nivel el cual tiene y cumple el estudiante como ser activo en su proceso de aprendizaje y formación, dentro de este se encuentran factores tales como cognición (estrategias de aprendizaje; memoria, solución de problemas), metacognición (planeación del desarrollo de la actividad), motivación (orientación a metas) y conductas. Este modelo se basa en la teoría socio-cognitiva de Bandura (1986), a partir de ello se postula que la autorregulación tiene tres factores los cuales intervienen en el funcionamiento del estudiante, estos son: personales encubiertos, conductuales y ambientales (Nocito, 2013).

Según Panadero y Alonso (2014), el modelo de Zimmerman plantea que la autorregulación del aprendizaje tiene como base el análisis de la actividad o tarea, este modelo plantea que existen tres fases:

a. Planificación: Mediante esta fase el estudiante analiza e interpreta la actividad a realizar y a partir de ello planifica la ejecución para llegar a cumplir la meta.

b. Ejecución: Mediante esta fase se ejecutan estrategias para llegar a la meta final de la tarea o actividad, con un interés y motivación.

c. Autorreflexión: Esta es la fase final durante esta el estudiante reflexiona sobre su proceso y experimenta emociones con relación a su capacidad de autorregulación. 


\section{Procesos de Autorregulación del aprendizaje}

Los procesos de aprendizaje, involucran las competencias, capacidades, conocimientos, estrategias metacognitivas, motivación, autoeficacia entre otras, las cuales permiten que el individuo se direccione hacia unas metas específicas de manera autónoma. A partir de ello, es importante, dentro de la formación académica tener en cuenta el proceso de Autorregulación del aprendizaje y la relación que tiene este con la enseñanza, puesto que mediante esta pueden desarrollarse y evidenciarse características particulares en los estudiantes.

De acuerdo con ello, Muchiut et al. (2018) afirma que la autorregulación del aprendizaje el estudiante, permite desarrollar un aprendizaje de manera autónoma, independiente, consiente, lo que permite que en el estudiante se genere un aprendizaje significativo, relacionando también los procesos tales como la autogestión, proactividad, autoconocimiento y autocontrol.

Estos procesos de autorregulación se encuentran en todas las modalidades de educación contemporánea, y niveles de educación, es decir, se puede evidenciar su desarrollo en estudiantes desde grados tales como primaria, hasta en estudiantes de educación superior, en las modalidades presencial, virtual y distancia tradicional. Para el desarrollo de estos procesos de autorregulación el rol del docente en el siglo XXI juega un papel fundamental, puesto que, mediante la práctica pedagógica y relación entre enseñanza - aprendizaje lo que se busca en generar en el estudiante una comprensión y aprendizaje autónomo. Ahora bien, con base en lo planteado anteriormente se procederá a describir tres de estos procesos de autorregulación, los cuales resultan pertinentes para el desarrollo de esta idea de investigación, dichos procesos son; la autoeficacia, el autoconcepto y la motivación.

\section{Autoeficacia}

El término de autoeficacia tiene sus bases en Bandura (1977), quien la definió como: "las creencias en la propia capacidad para organizar y ejecutar las acciones requeridas para manejar las situaciones futuras” (p. 2), con base en ello se considera que la autoeficacia hace referencia a la confianza que cada persona tiene de manera individual para lograr realizar alguna actividad (Canto, 2011). Esto supone que existe una relación directa y significativa, entre la autoeficacia y los logros académicos por parte del estudiante, puesto que, mediante el proceso de formación los estudiantes se plantean una meta de aprendizaje para regular y responder a su proceso académico, basados en un juicio sobre sus capacidades y la resolución de problemas.

\section{Autoconcepto}

De acuerdo con Véliz, Dörner y Sandoval (2016), el autoconcepto se comprende como un grupo de juicios sobre uno mismo, por medio de este se expresa y muestra el cómo la persona se percibe, conoce y representa; de allí se desprenden cuatro modelos; el nomotético, jerárquico, taxonómico y compensatorio. En relación con ello se han desarrollado investigaciones y aportes teóricos, sin embargo, el modelo que más teorías e investigaciones tiene es el modelo jerárquico, este postula el autoconcepto se encuentra relacionado con las dimensiones particulares del individuo. 


\section{Motivación}

La motivación en el proceso de autorregulación del aprendizaje juega un papel importante, puesto que, mediante ésta el individuo puede llegar a su meta establecida, con relación a ello se pueden evidenciar aportes a la motivación y el proceso de autorregulación, desde las teorías operante, fenomenológica, la teoría del procesamiento de la información, teoría sociocognitiva y teoría constructivista, en relación a ello Panadero y Alonso (2014), afirman que:

- Teoría del condicionamiento operante: comprende la motivación como una anticipación de las recompensas futuras, es decir de aquello positivo que se obtiene como resultado de la actividad.

- Teoría fenomenológica: la motivación se comprende como un factor que regula el comportamiento, relacionado de manera directa con la necesidad de sentirse incluido y activo.

- Teoría del procesamiento de la información (Johnson-Laird, 1988): esta postula que la actividad se ajusta en función de la información que el estudiante aprende en el momento.

- Teoría Sociocognitiva (Bandura, 1986 y 1997): esta teoría postula que el factor principal en relación a la autorregulación del aprendizaje son las metas y objetivos propios mediante ello la autoeficacia es percibida como un resultado.

- Teoría Constructivista, esta teoría realiza énfasis en el proceso cognitivo que desarrolla cada estudiante, buscando resolución de problemas.

\section{Autorregulación del aprendizaje en entornos virtuales}

La formación académica comprendida bajo los modelos distancia tradicional o virtual, comprende diversos factores, habilidades, competencias, con las cuales debe contar cada estudiante, docente y demás actores que hacen parte del contexto académico. Dichas habilidades y competencias las deben desarrollar no solo los estudiantes sino también los docentes que hacen parte de esta metodología, en estas habilidades se encuentran las relacionadas con las TIC, ya que, por medio de las herramientas tecnológicas, el estudiante puede de manera individual realizar una búsqueda, identificación y síntesis de la información relevante para su proceso académico, asimismo, permite potencializar un aprendizaje más amplio y crítico (García-Cabrero et al., 2018).

Las tecnologías de la información y comunicación en el ambiente educativo son consideradas como mediadores importantes en el proceso de enseñanza -aprendizaje, no solo en la modalidad virtual sino en todas las modalidades académicas, tales como presencial y distancia. De allí, que en muchas instituciones tanto y docentes durante sus clases, implementan laboratorios virtuales, audio libros, herramientas para mapas conceptuales, todo ello en pro de potencializar los conocimientos mediante otras herramientas diferentes a las tradicionales, a su vez, estas permiten acceder a la información de la manera más rápida y se pueden evaluar otras competencias.

En Colombia la educación virtual cuenta con un gran número de ofertas académicas y de estudiantes activos, los cuales de acuerdo con el Ministerio de Educación Nacional (MEN, 
2016) mostró un incremento del 98.9\%, asimismo, la educación virtual es una modalidad que involucra una visión en cuanto a las exigencias de tipo económico, social, político, y las TIC, es una perspectiva pedagógica en sí. Es decir que los programas académicos deben estar encaminados a desarrollar competencias en los estudiantes, las cuales les permitan desarrollar estrategias didácticas a través de las TIC, así, pues, se busca generar en cada uno de ellos un aprendizaje autónomo y autorregulado, desarrollar un compromiso académico y profesional.

\section{Rendimiento académico en estudiantes bajo la modalidad virtual}

El rendimiento académico es una variable de gran interés en el contexto académico, la cual se representa e implementa de manera cualitativa o cuantitativa, haciendo referencia a los logros y metas alcanzadas por un estudiante mediante su proceso académico, a su vez este resultado y evaluación puede ser individual o grupal, y en muchas oportunidades se asocia con la motivación al aprendizaje en un área específica. A partir de ello, se han realizado diversos aportes teóricos, definiciones y propuestas metodológicas para la evaluación del mismo constructo.

Algunos de estos autores son Vélez y Roa (2005), para quienes el concepto de rendimiento académico comprende procesos del estudiante tales como cognitivos y metacognitivos, autoestima, autoconcepto, autodeterminación, motivación, cada uno de ellos interviene de manera directa en el estudiante. Mediante este se puede evaluar lo aprendido por medio de un proceso de formación u enseñanza - aprendizaje.

Por otro lado, Jiménez (2000) sostiene que el rendimiento académico, hace referencia al conocimiento, capacidades, habilidades, representadas mediante sus actividades en las áreas académicas. De acuerdo con lo postulado por Albert Bandura, los estudiantes que no son auto eficaces en el aula, toda su atención y aprendizaje están en dirección a otras áreas como personales y sociales dejando a un lado su proceso académico.

\section{Factores que intervienen en el rendimiento académico}

Como se ha visto durante los párrafos anteriores, el rendimiento académico es una variable importante al momento de hablar sobre enseñanza - aprendizaje, asimismo mediante este proceso se pueden evidenciar diversos factores que intervienen en el rendimiento académico de un estudiante, estos factores pueden ser de tipo cognitivo, social, contextual. Con base en lo anterior se afirma que en el contexto académico es importante tener en cuenta que los factores que interviene en el proceso de formación del estudiante, puesto que el rendimiento académico es resultado de la interacción de estos (Martin citado en Mora, 2015).

Según Salinas, Bodensiek, Lucio y Molina (2010), existen variables y factores que influyen de manera directa en el rendimiento académico, estos se relacionan no solo con factores cognitivos, sino conductuales, emocionales y contextuales, estos son; edad, género, hábitos, experiencia académica, características familiares, nivel educativo de los padres, uso del tiempo, prácticas de crianza y relaciones de la familia con la escuela, entre otros. 


\section{Metodología}

Esta investigación, se desarrolló bajo un enfoque metodológico cualitativo con un diseño descriptivo. Se consideró dicho modelo puesto que este permite realizar énfasis en el significado y percepción de cada actor de la problemática a investigar. Se abordó esta metodología teniendo en cuenta que por medio de esta se buscó implementar estrategias que permitieran a los docentes favorecer y potencializar la autorregulación del aprendizaje en sus estudiantes durante la emisión de sus teleclases y de esta manera mejorar el rendimiento académico de los estudiantes en la asignatura de antropología social y cultural, además, este enfoque permite una planificación progresiva y analítica de investigación permitiendo abarcar el fenómeno en su totalidad. Tal y cómo sostiene, Maanen (citado en Álvarez-Gayou, 2013) este enfoque, comprende una serie de métodos y técnicas mediante las cuales se busca observar, describir, analizar y sintetizar el resultado de un fenómeno y de esta manera llegar a un resultado inductivo. Así pues, este enfoque permite conocer y analizar los diferentes procesos de autorregulación del aprendizaje en relación con el rendimiento académico desde un marco teórico práctico.

La población objeto de estudio estuvo constituida por trescientos estudiantes pertenecientes al programa de licenciatura en pedagogía infantil de modalidad virtual y docentes a cargo de dicho curso, en una institución universitaria de carácter privado, la cual tiene como misión contribuir al desarrollo sostenible, la convivencia y la paz, todo ello mediante el ejercicio de la docencia, la investigación y la proyección social con calidad. Para ello se conformó un grupo de interés de acuerdo con los objetivos, características e intereses de la presente investigación, es de tener en cuenta que se solicitó a los estudiantes hacer parte del estudio, para ello se seleccionó un grupo específico de 10 estudiantes, el cual se encontró constituido por 8 mujeres y 2 hombres, con edades comprendidas entre 17 y 42 años, quienes cursaron la asignatura Antropología social y cultural, durante el semestre anterior. Estos actores se encuentran ubicados en el departamento de Córdoba, Nariño, Santander, Antioquia (Colombia), dichos estudiantes se encuentran en municipios y veredas y dos docentes, el docente guía del curso y el acompañante de este. El enfoque cualitativo es fortalecido con un análisis estadístico de orientación cuantitativa para el procesamiento de la información obtenida.

Esta investigación se desarrolló bajo tres categorías o constructos los cuales son: estrategias de autorregulación del aprendizaje, niveles de desempeño académico y factores de autorregulación del aprendizaje que potencian el desempeño. Cada una de estas categorías contó con unas subcategorías las cuales conllevan a un análisis respectivo desde un enfoque metodológico cualitativo estas son: estrategias de aprendizaje, estrategias metacognitivas, resultados académicos, auto instrucción, autoevaluación, motivación e interés.

De acuerdo con Chavarría (2006), en la investigación educativa con paradigma cualitativo se implementan diversas técnicas de recolección de información dentro de estas se encuentran la observación y la entrevista, estas técnicas están orientadas y 
permiten conocer las experiencias de los participantes. A partir de ello, como instrumentos para la recolección de la información, evaluación y análisis de información para la investigación se implementaron dos instrumentos, el primero de ellos una entrevista semiestructurada dirigida y aplicada a los estudiantes, el segundo una encuesta abierta la cual fue diseñada, dirigida y aplicada a los docentes acompañantes de la asignatura.

Se realizó la validación de estos dos instrumentos por medio de juicio de expertos, esto con el objetivo de generar confiabilidad y objetividad en los instrumentos, este juicio de expertos fue realizado por dos docentes con alta experiencia en investigación en educación, quienes a partir de una revisión determinaron la claridad y coherencia de los instrumentos teniendo en cuenta los indicadores y los objetivos de cada uno de los ítems. Una vez organizado el grupo de interés y validación de expertos correspondiente de instrumentos, se realizó una presentación con los actores donde se explicó el objetivo de la investigación, el instrumento que se utilizaría, se envió el consentimiento informado, todo ello mediante el correo electrónico institucional, asimismo se acordó la fecha y hora para la entrevista la cual se realizó vía telefónica ya que, no fue posible establecer otro canal de comunicación, donde se realizó una introducción breve nuevamente sobre el objetivo de la investigación y confidencialidad de la información.

\section{Resultados}

La información fue analizada y sistematizada mediante tres categorías: Estrategias de autorregulación del aprendizaje, Niveles de desempeño académico y Factores de autorregulación del aprendizaje que potencian el desempeño académico.

\section{Estrategias de Autorregulación del aprendizaje}

Se consideraron las estrategias son utilizadas e implementadas por los estudiantes, al momento de realizar la lectura del material disponible en los módulos de la asignatura. Los resultados se evidencian en la Tabla 1 y Tabla 2.

TABLA 1.

Estrategias de autorregulación del aprendizaje

\begin{tabular}{|c|c|c|}
\hline Estrategia & Instrumento & Descripción del hallazgo \\
\hline $\begin{array}{l}\text { Estrategias de } \\
\text { aprendizaje }\end{array}$ & $\begin{array}{l}\text { Entrevista } \\
\text { semiestructurada }\end{array}$ & $\begin{array}{l}\text { Se lograron identificar las estrategias de aprendizaje } \\
\text { de identificación e inferencia de texto, verbalización y } \\
\text { comprensión del texto y releer y evaluar el texto, las cuales } \\
\text { los estudiantes utilizan para la realización de las lecturas y } \\
\text { material disponible en la plataforma virtual. }\end{array}$ \\
\hline $\begin{array}{l}\text { Estrategias Meta } \\
\text { cognitivas }\end{array}$ & $\begin{array}{l}\text { Entrevista } \\
\text { semiestructurada }\end{array}$ & $\begin{array}{l}\text { Se identificó el nivel de Conocimiento, autonomía, } \\
\text { planificación, ejecución y control , conocimiento y ejecución, } \\
\text { en relación a la planificación y control del tiempo de los } \\
\text { estudiantes con relación a su proceso académico. }\end{array}$ \\
\hline
\end{tabular}

Fuente: Elaboración propia. 
TABla 2.

Estrategias de aprendizaje implementadas por los estudiantes

\begin{tabular}{|c|c|}
\hline Estrategia & Descripción del hallazgo \\
\hline $\begin{array}{l}\text { Identificación } \\
\text { e inferencia } \\
\text { del texto }\end{array}$ & $\begin{array}{l}\text { La identificación de los temas principales de una lectura, se da a partir de diversas } \\
\text { herramientas de inferencia, la cuales se implementan como estrategia el momento de } \\
\text { desarrollar una lectura: según lo referido por los estudiantes estas suelen ser: sacar ideas } \\
\text { principales, tomar apuntes, sacar conclusiones y realizar resúmenes. }\end{array}$ \\
\hline $\begin{array}{l}\text { Verbalización } \\
\text { y } \\
\text { comprensión } \\
\text { del texto }\end{array}$ & $\begin{array}{l}\text { La verbalización o lectura "en voz alta" plantea una asimilación frente a la oralidad, la } \\
\text { cual facilita la comprensión del texto, en relación a ello, los estudiantes sostienen que como } \\
\text { estrategia durante la lectura de textos realizan lectura en voz alta ya que, esto les permite } \\
\text { tener una mayor comprensión del texto y por ente un aprendizaje. }\end{array}$ \\
\hline $\begin{array}{l}\text { Releer el } \\
\text { texto y } \\
\text { evaluación } \\
\text { del texto }\end{array}$ & $\begin{array}{l}\text { Realizar una lectura del texto varias veces e implementar una evaluación critica del mismo, } \\
\text { conllevan a una comprensión del texto desde una postura analítica, de acuerdo con los } \\
\text { estudiantes la estrategia de releer el texto se considera una estrategia importante ya que, } \\
\text { permite comprender el texto una y otra vez, por ende queda en total claridad los temas } \\
\text { abordados. }\end{array}$ \\
\hline
\end{tabular}

Fuente: Elaboración propia.

El 50\% de los estudiantes reconocen que, para lograr comprender los textos y material disponible en la plataforma, implementan la identificación e inferencia de texto, ya que, esta estrategia les es útil. Por otro lado, los estudiantes implementan otras estrategias dentro de las cuales se encuentran; la verbalización y comprensión, en esta estrategia los estudiantes argumentan que, para la lectura de textos y comprensión de estos, deben leer en voz alta realizando una oralidad de los mismos, tal y como lo sostiene el estudiante.

\section{Estrategias Metacognitivas}

Se buscó identificar una autonomía, planificación y control frente a el tiempo y horario establecido para el desarrollo de actividades académicas por parte de los estudiantes ya que, estas son importantes para el desarrollo de un aprendizaje autorregulado. Esto se evidencia en la Tabla 3.

TABLA 3.

Estrategias Metacognitivas

\begin{tabular}{ll}
\hline \multicolumn{1}{c}{ Estrategia } & \multicolumn{1}{c}{ Descripción del hallazgo } \\
\hline $\begin{array}{l}\text { Conocimiento, } \\
\text { autonomía, } \\
\text { planificación, } \\
\text { ejecución y }\end{array}$ & $\begin{array}{l}\text { Dentro de las estrategias metacognitivas, el estudiante debe contar con un conocimiento } \\
\text { o autoconciencia de sus procesos de aprendizaje, asimismo de lo que requiere para la } \\
\text { ejecución de sus actividades, a partir de ello y de manera autónoma realiza una reflexión y } \\
\text { planificación de sus actividades, estimando y ejecutando un tiempo para su aprendizaje y } \\
\text { control del mismo, de esta manera podrá supervisar, comprobar sus procesos y estrategias } \\
\text { metacognitivas para alcanzar sus metas y objetivos de aprendizaje. }\end{array}$ \\
& $\begin{array}{l}\text { Existe un reconocimiento de las estrategias metacognitivas las cuales son importantes } \\
\text { para el desarrollo y adquisición de un aprendizaje y ejecución de actividades, sin embargo, } \\
\text { debido a factores externos no se desarrollan ni ejecución el tiempo para el desarrollo e } \\
\text { implementación de estas, llevando de esta manera a un conocimiento y ejecución inmediata } \\
\text { ejecución }\end{array}$ \\
& de las actividades.
\end{tabular}

Fuente: Elaboración propia. 
Los estudiantes, cuentan con estrategias de meta cognición establecidas, dedicando alrededor de dos horas diarias, para el conocimiento, autonomía, planificación, ejecución $y$ control, de sus actividades académicas y proceso de aprendizaje. Por otro lado, los estudiantes implementan estrategias de conocimiento y ejecución de manera directa de las actividades académicas, esto se debe a que no cuentan con un horario establecido diario.

Niveles de desempeño académico (Tabla 4)

TABLA 4.

Escala valorativa de importancia del proceso académico, desempeño y resultados

\begin{tabular}{|c|c|c|c|}
\hline Estudiante & $\begin{array}{c}\text { Importante porque lleva a } \\
\text { un aprendizaje }\end{array}$ & $\begin{array}{l}\text { Importante lleva a una } \\
\text { aprobación de cursos }\end{array}$ & $\begin{array}{c}\text { Importante, el profesor es la } \\
\text { guía }\end{array}$ \\
\hline$E_{1}$ & & $X$ & \\
\hline$E_{2}$ & & $X$ & \\
\hline$E_{3}$ & & $x$ & \\
\hline$E_{4}$ & & $x$ & \\
\hline$E_{5}$ & & $X$ & \\
\hline$E_{6}$ & & $X$ & \\
\hline $\boldsymbol{E}_{7}$ & $X$ & & \\
\hline$E_{8}$ & $X$ & & \\
\hline$E_{9}$ & & & $X$ \\
\hline$E_{10}$ & $X$ & & \\
\hline
\end{tabular}

Fuente: Elaboración propia.

A partir de esta escala valorativa, se puede evidenciar que, las notas que tienen como resultado de su proceso académico las asocian de manera directa con la aprobación de los cursos y valoración cuantitativa para un promedio final. Para menos de la mitad de la muestra, el rendimiento académico y las notas obtenidas no solo son importantes, sino que las asocian de manera directa con el proceso mediante el cual les permite tener un aprendizaje significativo.

Factores de autorregulación del aprendizaje que potencian el desempeño académico

\section{Motivación}

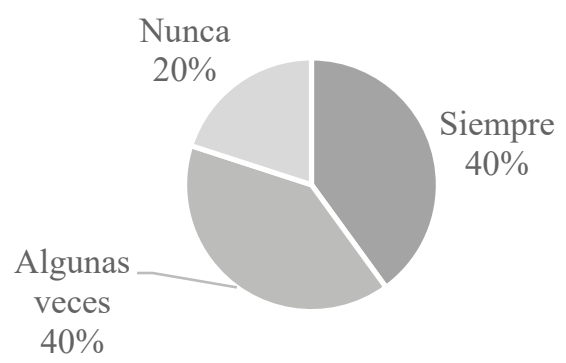

Figura 1. Nivel de participación mediante foros virtuales Fuente: Elaboración propia. 
A los estudiantes les motiva la posibilidad de compartir un espacio de saberes y debate con el resto de sus compañeros a nivel nacional, así pues, se desataca la Participación siempre en los foros de debate durante la clase, o activa durante las teleclases, No obstante, en un mismo porcentaje de estudiantes correspondientes a la muestra, manifiestan que su participación no es activa o constante por lo que se destacó la variable Participa algunas veces en los foros de debate durante la clase I (Figura 1).

\section{Resultados entrevista a docentes}

El acompañamiento y la realimentación contante por pare del docente, se relaciona de manera directa con los factores motivaciones en los estudiantes (Tabla 5).

TABLA 5.

Acompañamiento y estrategias motivaciones por parte de docente tutor y acompañante

\begin{tabular}{ll}
\hline Docente & \multicolumn{1}{c}{ Resultados y Descripción del hallazgo } \\
\hline & $\begin{array}{l}\text { Niveles de rendimiento bajo, falta reflexión y compromiso frente a los procesos } \\
\text { pedagógicos }\end{array}$ \\
& $\begin{array}{l}\text { De acuerdo con los resultados académicos en el curso, denota que los estudiantes dedicaron un } \\
\text { tiempo mínimo, los estudiantes requieren demostrar y desarrollar compromiso y responsabilidad } \\
\text { con su proceso académico. }\end{array}$ \\
& $\begin{array}{l}\text { Niveles de rendimiento bajo, incipiente referencias teóricas y revisión de material } \\
\text { pedagógico }\end{array}$ \\
& $\begin{array}{l}\text { A partir de un análisis, comparación e identificación de debilidades se puede establecer que } \\
\text { las entregas de actividades académicas muestran una incipiente referencia de lecturas, los } \\
\text { estudiantes deben ampliar su investigación y revisión de material para la elaboración de } \\
\text { actividades. }\end{array}$ \\
\hline
\end{tabular}

Fuente: Elaboración propia.

Con base en la tabla anterior, se puede evidenciar que las prácticas y estrategias que implementan los docentes para potencializar la motivación en los estudiantes del curso antropología social y cultural, se encuentran brindar un acompañamiento constante y permanente mediante los diversos medios de comunicación.

\section{Estrategias pedagógicas}

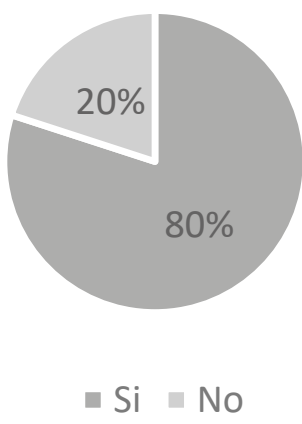

Figura 2. Escala metodología de la clase de antropología social y comprensión y el aprendizaje Fuente: Elaboración propia. 
La Figura 2 muestra que más de la mitad del grupo consideran que la metodología y procesos pedagógicos llevados a cabo por el docente durante la clase, permitieron una comprensión y un aprendizaje durante el curso de antropología social y cultural, y en un porcentaje inferior algunos estudiantes consideran que esta metodología no proporciono o potencializado alguna comprensión o aprendizaje alguno, durante el curso y semestre.

\section{Resultados entrevista a docentes}

En esta subcategoría se tienen en cuenta las estrategias pedagógicas que establecieron los docentes para lograr un aprendizaje significativo en los estudiantes de la asignatura de antropología social y cultural (Tabla 6).

TABLA 6.

Estrategias pedagógicas por docente

\begin{tabular}{|c|c|}
\hline Estrategia & Resultados \\
\hline $\mathrm{D}_{1}$ & $\begin{array}{l}\text { Estrategias pedagógicas enmarcadas desde el saber } \\
\text { Se presentaron estas estrategias mediante la plataforma de aprendizaje virtual y teleclases, } \\
\text { todas enfocadas desde el saber, saber ser y saber hacer. }\end{array}$ \\
\hline$D_{2}$ & $\begin{array}{l}\text { Estrategias pedagógicas Aprovechamiento de recursos } \\
\text { Se potencializa en la plataforma virtual estrategias desde una planeación de contenido, } \\
\text { con contenidos orientados al aprovechamiento de los recursos virtuales, en los módulos, } \\
\text { bibliotecas. }\end{array}$ \\
\hline
\end{tabular}

Fuente: Elaboración propia.

\section{Autoeficacia}

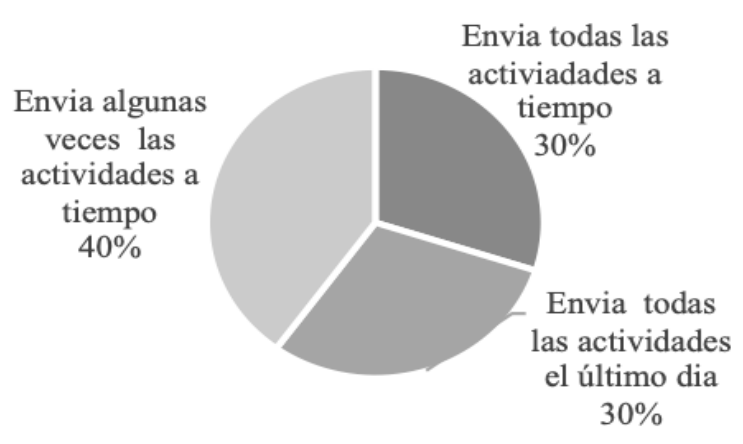

Figura 3. Tiempo de envío de actividades Fuente: Elaboración propia.

En la Figura 3 se muestran los porcentajes y variables de tiempo de realización y envío de actividades académicas, asimismo se puede evidenciar que menos de la mitad de los estudiantes afirman enviar sus actividades académicas algunas veces a tiempo, frente a ello los estudiantes sostienen que esto se debe a factores externos relacionados con las TIC y falta de tiempo por sus actividades laborales y personales. Asimismo, en un porcentaje inferior, los estudiantes sostienen enviar todas sus actividades con tiempo suficiente. 


\section{Resultados de la encuesta}

El docente y rol de éste dentro del proceso pedagógico es importante ya que, por medio de la mediación del docente genera mejores resultados en su proceso de aprendizaje y autoeficacia (Figura 4).

\section{Reflexión pedagógica}

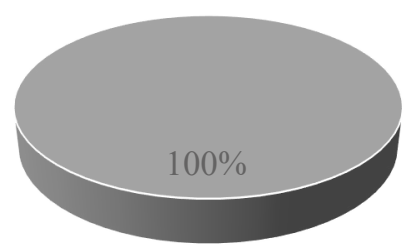

Figura 4. Porcentaje de la Estimulación de Reflexión pedagógica en estudiantes Fuente: Elaboración propia.

De acuerdo con los docentes, durante el proceso de enseñanza- aprendizaje se desarrollaron espacios de retroalimentación, los cuales promueven una reflexión pedagógica en los estudiantes, sin embargo, los docentes expresan que, para dar continuidad a ello, se presentan algunas dificultades que no permiten la atención, realimentación y reflexión pedagógica constante en la asignatura de antropología social y cultural.

\section{Discusión y Conclusiones}

Los resultados de la investigación permiten determinar, que los estudiantes cuentan con una serie de estrategias de aprendizaje y estrategias metacognitivas, las cuales implementan de manera individual durante su proceso pedagógico, permitiéndoles desarrollar la lecturas y verificación de material correspondiente a la asignatura y de esta manera no tener solo una identificación, sino una comprensión y posteriormente una evaluación de su aprendizaje.

La investigación permitió evidenciar que, los estudiantes reconocen la importancia de los niveles obtenidos como resultado de su proceso de aprendizaje y pedagógico, sin embargo, para algunos estudiantes las notas como resultado de su proceso académico están asociadas de forma directa con la aprobación de los cursos y valoración cuantitativa para un promedio final, dejando a un lado el aprendizaje logrado, a diferencia de los que comprenden esos niveles como un proceso importante para su formación profesional y aprendizaje, confirmando así lo que sostienen Vélez y Roa (2005) cuando asocian el rendimiento académico de manera directa con los procesos cognitivos, de autoestima y motivación en el estudiante y de esta manera se evalúa el proceso de enseñanza - aprendizaje.

Sé logró establecer que los estudiantes cuentan con una motivación por su proceso de aprendizaje, por ello participan durante sus teleclases, ya que, consideran que este espacio es de gran valor, puesto que pueden exponer sus ideas e intercambiar saberes con los compañeros a nivel nacional. Asimismo, los docentes generan estrategias de acompañamiento que potencian esta motivación, sin embargo, debido a las dificultades de conectividad en muchas oportunidades esta participación es nula. Durante las teleclases, se desarrollan 
estrategias pedagógicas, las cuales se comprenden como una herramienta importante puesto que, permiten a los estudiantes la adquisición de un aprendizaje significativo; según los estudiantes, se encuentran los videos pedagógicos y material lúdico, y desde la perspectiva de los docentes se encuentran las estrategias enmarcadas desde el saber y el aprovechamiento de recursos, relacionándose así como lo que sostiene el MEN (2016), donde la educación virtual implica una visión de las exigencias de los diversos entornos, tanto económico, como social y político, buscando una educación de calidad.

En cuanto a los procesos de autoeficacia, se evidenció que, aunque los docentes generan un acompañamiento permanente buscando una reflexión pedagógica constante, los estudiantes suelen enviar sus actividades cerca a la fecha de cierre del sistema, de acuerdo con las narraciones de los estudiantes, esto se debe relacionado a factores externos tales como la conectividad, tiempo y ocupaciones personales, sin embargo, no cuentan con una estrategia la cual les permita poner superar este obstáculo, ya que esto es importante en el proceso de autoeficacia en el estudiante, tal y como sostiene Alegre (2014), las perspectivas que tiene el estudiante en relación a su autoeficacia se relaciona con la actividad y desarrollo de sus actividades, de esta manera gestiona estrategias que le permiten llegar a una meta establecida.

A partir de los hallazgos, se puede concluir que existe una relación entre los procesos de autorregulación del aprendizaje tales como motivación, autoeficacia, autoconcepto y el desempeño académico en los estudiantes de licenciatura en pedagogía infantil, puesto que, se mostró que los estudiantes que cuentan con hábitos de estudio tales como un tiempo determinado diario para la realización de sus actividades académicas, uso de estrategias de autorregulación, muestran mayor confianza frente a su aprendizaje autónomo y por ende su rendimiento académico, a diferencia de los que por diversos factores no cuentan con hábitos de estudio establecidos y uso de estrategias.

Sin embargo, se identificó que un número de estudiantes no cuenta con procesos de autorregulación establecidos, lo cual genera dificultad no solo en su proceso de aprendizaje, sino en su desempeño académico, con base en ello, es importante el fortalecimiento de un aprendizaje el cual le permita al estudiante fortalecer su autoeficacia, habilidades y hábitos de estudio, ya que esto le permite establecer metas claras en su proceso académico, tener una confianza en sí mismo e identificar posibles obstáculos que se presentan y poder sobresalir de ellos.

\section{REFERENCIAS}

Alegre, A. (2014). Autoeficacia académica, autorregulación del aprendizaje y rendimiento académico en estudiantes universitarios iniciales. Propósitos y representaciones, 2(1), 79-120. http://dx.doi.org/10.20511/pyr2014.v2n1.54

Álvarez-Gayou, J. (2013). Como hacer una investigación cualitativa. Fundamentos y metodología. México, D.F.: Paidós.

Bandura, A. (1977). Self-efficacy: Toward unifying theory of behavior change. PsychologicalReview, 84(2), 191-215. http://dx.doi.org/10.1037/0033-295X.84.2.191

Canto, J. E. (2011). Autoeficacia y educación. Educación y ciencia, 2(18), 45-53. Disponible en http://www.educacionyciencia.org/index.php/educacionyciencia/article/view/142 
De Acedo, M. (2010). Competencias cognitivas en educación superior. Madrid: Narcea. http:// dx.doi.org/10.4995/redu.2013.5541

García-Cabrero, B., Serrano, L., Cisneros, E., Arroyo, C., Díaz, E. y Vigil, M. H. (2018). Las competencias docentes en entornos virtuales: Un modelo para su evaluación. Revista Iberoamericana de Educación a Distancia, 21(1), 343-365. http://dx.doi.org/10.5944/ ried.21.1.18816

Hernández, A. y Camargo, Á. (2017). Autorregulación del aprendizaje en la educación superior en Iberoamérica: una revisión sistemática. Revista Latinoamericana de Psicología, 49(2), 146-160. https://doi.org/10.1016/j.rlp.2017.01.001

Jiménez, M. (2000). Competencia social: intervención preventiva en la escuela. Revista Infancia y Sociedad, (24), 21-48. Disponible en https://dialnet.unirioja.es/servlet/ articulo?codigo $=4353980$

Klimenko, O. y Alvares, J. L. (2009). Aprender cómo aprendo: la enseñanza de estrategias metacognitivas. Educación y educadores, 12(2), 11-28. Disponible en https://personaybioetica.unisabana.edu.co/index.php/eye/article/viewFile/1483/1652

Mora, R. T. (2015). Factores que intervienen en el rendimiento académico universitario: Un estudio de caso. Opción, 31(Especial 6), 1041-1063 Disponible en http://hdl.handle. net/10045/52320

Moreno, O. (2015). Evaluación de la modalidad de interacción de la tutoría y los efectos en logro académico en entornos en línea. RIED: Revista Iberoamericana de Educación a Distancia, 18(1), 231-255. Disponible en http://e-spacio.uned.es/fez/eserv/bibliuned:revi staRied-2015-18-1-7110/evaluacion_modalidad.pdf

Muchiut, Á. F., Zapata, R. B., Comba, A., Mari, M., Torres, N., Pellizardi, J. y Segovia, A. P. (2018). Neurodidáctica y autorregulación del aprendizaje, un camino de la teoría a la práctica. Revista Iberoamericana de Educación, 78(1), 205-219. https://doi.org/10.35362/ rie7813193

Nocito, G. (2013). Autorregulación del aprendizaje de alumnos de grado. Estudio de caso [Doctoral dissertation]. Universidad Complutense de Madrid, Madrid, España.

Panadero, E. y Alonso-Tapia, J. (2014). Teorías de autorregulación educativa: una comparación y reflexión teórica. Psicología educativa, 20(1), 11-22. https://doi.org/10.1016/j. pse.2014.05.002

República de Colombia. MEN. (2016). Educación virtual o educación en línea. [Online]. Disponible en https://www.mineducacion.gov.co/1759/w3-article-196492.html?_noredirect=1

Salinas, F., Bodensiek, A., Lucio, R. y Molina, A (2010). Estudio sobre los Factores que influyen en el rendimiento escolar. Bogotá, D.C.: Secretaria de Educación Distrital. [Online]. Disponible en https://evaluacion.educacionbogota.edu.co/files/Factores\%20que\%20 influyen $\% 20 \mathrm{en} \% 20 \mathrm{el} \% 20$ rendimiento\%20escolar.pdf

Vélez, A. y Roa, C. N. (2005). Factores asociados al rendimiento académico en estudiantes de medicina. Educación médica, 8(2), 24-32. https://doi.org/10.4321/S157518132005000200005

Véliz, A., Paris, A. y Sandoval, S. (2016). Relacion entre autoconcepto, autoeficacia académica y rendimiento académico en estudiantes de salud de Puerto Montt, Chile. Educadi, 1(1)97-109. Disponible en http://portalrevistas.uct.cl/index.php/educadi/article/ view/1003 
Claudia Tatiana Mora Yate es psicóloga, maestrante en educación (proceso de grado) de la Corporación Universitaria Minuto de Dios (Colombia). Programas de prevención y promoción de consumo de sustancias psicoactivas. Manejo de plataformas virtuales de aprendizaje Moodle y Blackboard. Competencias ofimáticas y manejo de herramientas TIC (herramientas Web 2.0). https://orcid.org/0000-0001-7766-9392

Jenny Consuelo Mahecha Escobar es magister y especialista en comunicación educativa, trabajadora social con experiencia en docencia universitaria en las áreas de formación humana y comunicación escrita y procesos lectores por más de 8 años. Acompañamiento de estudiantes en educación virtual, y presencial; asesoría en procesos pedagógicos, fortalecimiento de los procesos y estrategias de enseñanza-aprendizaje en educación bajo modalidad presencial y virtual. Actualmente se desempeña como docente-tutora de proyectos de investigación aplicada i, ii y iii bajo la línea de investigación: autorregulación en el aprendizaje, además de los cursos: tecnología e innovación en la educación y teorías y estilos de aprendizaje y líder del curso inclusión educativa de la maestría en educación de la Uniminuto (Colombia). https://orcid.org/0000-0002-9799-7200

Francisco Conejo Carrasco es doctorando en Investigación Transdisciplinar en Educación de la Universidad de Valladolid (España). Licenciado en Historia de misma universidad y especialista en Historia Moderna, Contemporánea y de América. Máster en Profesorado de Educación Secundaria Obligatoria y Bachillerato, Formación Profesional y Enseñanza de Idiomas, por la misma universidad. Docente-investigador desde hace más de cinco años. Ha desempeñado su labor docente en el marco de la investigación como en los cursos: Introducción a la investigación educativa del Máster en Profesorado de Educación Media, de la Universidad de Valladolid (España), docente-tutor en la Maestría en Educación con el Tecnológico de Monterrey (México)-UNIMINUTO, impartiendo las siguientes asignaturas: Gestión de Ambientes de Aprendizaje, Educación para el Desarrollo, Tecnología e Innovación en la Educación, Teorías y estilos de aprendizaje y las materias de acompañamiento en investigación aplicada: PIA I, PIA II y PIA III (2013 - Actualidad). También ha trabajado como investigador becado en el Proyecto TRADDEC I+D+i del Ministerio de Economía y Competitividad del Gobierno del Reino de España (2010-2014). Se ha desempeñado como profesor de la asignatura transversal: Proyecto de Vida en la Corporación Universitaria Minuto de Dios - UNIMINUTO, Sede Calle 80 presencial (2014-2018). También como Coordinador de Formación Humana en la Vicerrectoría Regional Bogotá Sur (2019-actualidad). Actualmente desarrolla la línea de investigación: Autorregulación en el aprendizaje de la Maestría en Educación de UNIMINUTO, trabajando como Docente líder de la Maestría en las asignaturas: Teoría y estilos de aprendizaje, PIA I, PIA II y PIA III (2017-actualidad). Pertenece al Grupo de Investigación de UNIMINUTO Virtual y a Distancia (GIUVD), categorizado B en COLCIENCIAS. Investigador JUNIOR. https://orcid.org/0000-00023490-1937 